$\xi=1$ 圆

\title{
Smart city ambulance for tracking shortest path using global position system
}

\author{
Dr.M. Prakash*, S. Nithyanantham, V. Nishanth ,A. Prakash, D. Kaviyarrasu \\ Department of Computer Science and Engineering, Coimbatore \\ Corresponding author: salemprakash@gmail.com
}

\begin{abstract}
Traffic overcrowding and tidal flow management were identified as major problems in modern urban areas, which have caused much uncomfortable for the ambulance. Moreover, road accidents in the city have been nonstop and to bar the loss of life due to the accidents is even more crucial. To implement this, we introduce a scheme called Smart city ambulance system using shortest path finding algorithm and traffic signals. The main theme behind this scheme is to provide a smooth flow for the ambulance to reach the hospitals in time and thus minifying the expiration. The ambulance driver will send the request to control room. After receiving the request from control room then the ambulance is controlled by the control room which furnishes the most scant route to the ambulance and also controls the traffic light according to the ambulance location and thus reaching the hospital safely. The control room will send the alert message to the hospital. This scheme is fully controlled by control room, thus it controls the traffic lights, helping to reach the hospital in time using the shortest path Dijkstra algorithm
\end{abstract}

Keywords: IoT, Smart Ambulance devices, Ultra Sonic Sensor, GPS (global position System), Ambulance, Emergency

\section{Introduction}

Today's problem, there are many cities which are working on transforming themselves into Smart Cities. If the city is going to be called as Smart City, then it should have all possible advancements in the sector of smart technology. Improving efficiency in healthcare sector is one of the difficult and most challenging jobs. That includes various aspects such as getting ambulance within minimum amount of time, providing proper treatment to the patient so that the chances of surviving increases in critical condition. Traffic congestion is one of the major problems in urban areas, which have caused much hitches for the ambulance. Moreover, road accidents in the city have been increased and to bar the loss of life due to the accidents (or) ambulance is stuck in traffic signal is even more crucial. We can overcome traffic signal problems and choosing the shortest path to reach the hospital as soon as possible using control room. The ambulance driver will send the request to the control room. And control room will search the shortest path and hospital. And the control room will send the alert message to the hospital and also the control room will send the shortest path to the ambulance driver. It is also an attempt to participate actively in the process of transforming into smart city and make required services more accessible.

\section{Background and Related work}

An ambulance vehicle which is used to transist of injured people or sick to from or between places of providing medical treatment to rescue their life and in some certain situation that will make a instance to provide out of hospital medical care to the patient.

Google has developed API that is Google Map which can be used to find out the nearest hospital which is located to the same route and it will display only the available hospitals here the drawbacks are while going to the hospital ambulance may possible to stuck into traffic congestion area so what here we are done we will designed to display the detailed about the non-traffic area and it will display only the nearest hospital and also without any road congestion. If ambulance driver will chose the wrong way to reach the hospital. Patient will get in critical situation or else the necessary equipment will not present in the hospital, the ambulance driver will going to another hospital. During that situation drive may do not know the route. Here this application can be helpful for the drive.

In Precious time in these kind of emergencies is when the patient enters the ambulance and the driver will pick the patient for hospital. After reaching the hospital the doctor will check the patient condition if the condition is good after that the management will make arrangement for patient during these process the patient will go for serious condition. This paper will avoid the serious condition because the ambulance will give the alert signal to the hospital. And hospital will make arrangement for patient's condition

The Importance of communication during the disaster times is understood well by this project. Emergency situation includes disasters like fire, medical emergencies, accidents, earthquake, floods or any other natural calamity. The project works on enabling ad hoc smart phone based communications at the emergency time over WIFI, to avail the service the person in trouble shall call EMS(Emergency Management System) designed by this project. Requests are received by the server. Lent server system principle is used the server 
responds to the client's request. Client and rescue application is developed as an android application. Server is implemented as a web based application. The system is tested using various GPS enabled android Phone.

The objective of this paper is to discuss a general reference framework for the design of an urban IoT. We describe the specific characteristics of an urban IoT, and the services that may drive the adoption of urban IoT by local governments. We then overview the web-based approach for the design of IoT services, and the related protocols and technologies, discussing their suitability for the Smart City environment.

\section{Proposed System}

As discussed in the drawbacks section of the existing procedures in ambulance services, improving the ambulance service efficiency in healthcare sector is difficult task. But a smart city should provide good services in all the sectors including effective traffic management system during emergency periods. Even many public sectors require solutions and possibilities to improve its services, healthcare sector and emergency vehicle services require first preference. With this motivation we are proposing a scheme called Smart city ambulance system using shortest path finding algorithm on available paths and managing traffic signals. The main objective of our model is to provide a smooth traffic free path for the ambulance to reach the hospitals in time and thus saves the patients. to avoid the traffic delay on travelling which provides fast services to any vehicles. To find the shortest path as well as traffic free route to any vehicles that requires emergency service. To manage the traffic signals based on the current traffic and provide effective city traffic management.

To establish the proper communication channel between the control rooms and vehicles that requires emergency service. To provide the facilities for vehicles to enrolled in this smart application which avoids the traffic.

\section{Methods and Materials}

\section{Explanation:}

Before picking up the patient the ambulance driver will give the signal to control room. In control room they will search the nearest hospital and find the shortest path to reach hospital. From security cameras the control room will calculate the traffic percentage and then find the shortest path to reach a hospital and in first shortest path traffic percentage according to the level of traffic congestion path will be suggest to the ambulance driver then give a green signal for the shortest path and block (or) give red signal for other roads because the other road signal is disturbing the first shortest path. After passing of ambulance on a specific signal, the signal will act normally. Control room will send the messages for both ambulance and hospital Ambulance will receive the shortest path form control room. Hospital will receive the alert message (or) warning alarm from the control room. The ambulance driver can carry a patient and easily reach the hospital with in a time. And hospital also prepare for emergency ward. It will save more people's life.

\section{Option:1}

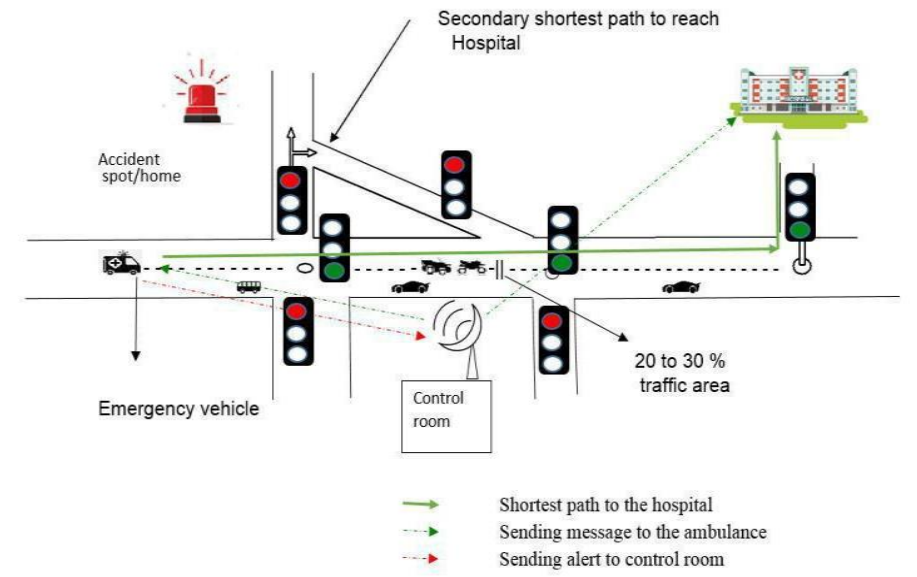

Fig 3.1: Low Level Traffic.

In this module by the way of measuring mechanism to find the traffic percentage if it is $20-30 \%$ of congestion occurred the method fig 3.1 will be the suitable for to reach the hospital.

\section{Option 2:}

In this module if it is $50-60 \%$ of traffic will occur as shown in fig 3.2 control room will suggest the alternate path to reach the hospital by the way of block the one route and provide the path for the ambulance.

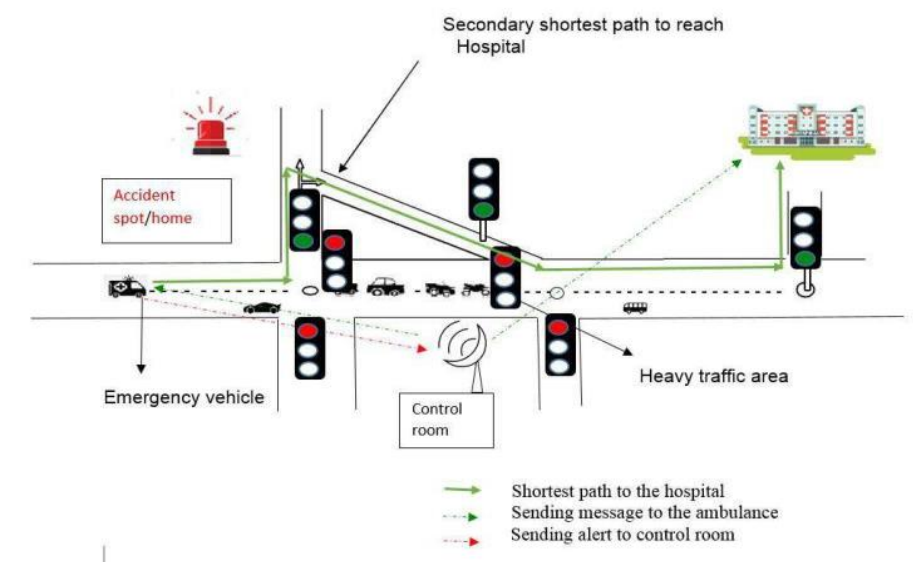

Option: 3

Fig 3.2: Medium Level Traffic.

In this module it is suitable for $60 \%$ and above traffic percentage and also shortest path is four-way road then we have added the new take over signal and free one way for

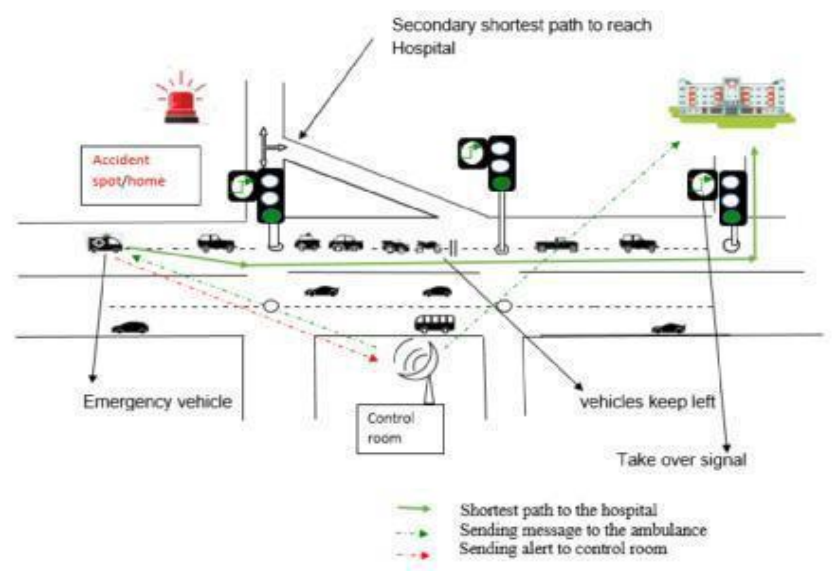

Fig 3.3: Highway Level Traffic 
ambulance and another way for normal vehicle. In this method is not affect the public and ambulance also.

But it required law when the normal vehicle drivers see this signal after within a minute to give the right side way to the ambulance. This method can easily to help the ambulance to reach the hospital and people also didn't affect and the control room can give the takeover signal for public between the accident spot to hospital. Control room will send the messages for both ambulance and hospital. Ambulance will receive the shortest path form control room. Hospital will receive the alert message (or) warning alarm from the control room. The ambulance driver can carry a patient and easily reach the hospital with in a time. And hospital also prepare for emergency ward. It will save more peoples of life.

\section{Design and Implementation}

The implementation of this system is divided into front end as well as back end. In that front end is nothing but a ambulance which is connect to the control room through the server and it will suggest the appropriate nearest hospital and the server side used for handling ambulance driver requests and respond by processing them.

\section{Client end:}

This application will initially be based on GPS by the way of locating the current location which is in the accident spot or patient home after that it will send the request to control room and it will check the nearest hospital based on the time and also it will send pre-alert to the hospital .This query is sent in the JSON format to the server. Server will process on it and respond accordingly. The response from server will also be in JSON format and read the data from control room.

\section{Server end:}

Server end it act as admin and mainly used for instructing the route to the hospital. When server will receive a request from ambulance driver and also it will list out nearest hospital based on the patient illness and time efficiency is a primary concern, it will extract result from the database .for every request it will send response using the JSON in the part of POST/GET method.

\section{JSON (JavaScript object notation)}

It helps the application to sending and receiving the information from the database. we can store the data or information in a organized manner.it helps to transfer the data from front end to back end. It is in text format and this helps in computing language independency. Hence, it works as an ideal tool. It has 2 basic same parts namely

$$
\begin{array}{ll}
\text { i. } & \text { Key } \\
\text { ii. } & \text { Value }
\end{array}
$$

Where they both together are used as a pair In JSON, the key denotes a string which needs to be enclosed between quotation marks, whereas values in the form of string, numbers, array and Boolean expression or it can be an object.

\section{Why use JSON}

Simplicity:

JSON is easy to understand and smallegrammer then XML. JSON has better capability for mapping onto the data structure than XML.

Extensipility:
Comparatively, JSON has a better and simpler structure compared to XML and is more easy and faster to process. Even though software is available for the programmer to handle XML, some additional software or code is required. Access rate of JSON is faster than XML as it uses fewer words compared to XML.

\section{Mathematical Model:}

Let consider $\mathrm{H}, \mathrm{A}, \mathrm{U}, \mathrm{S}$ are the variables .

$\mathrm{H}$ denotes the Number of Hospitals

$\mathrm{H}=\{\mathrm{h} 1, \mathrm{~h} 2, \mathrm{~h} 3 \ldots \ldots \ldots . \mathrm{hn}\}$

A - Denotes the Number of Hospitals:

$\mathrm{A}=\{\mathrm{a} 1, \mathrm{a} 2, \mathrm{a} 3 \ldots \ldots \ldots \mathrm{an}\}$

$\mathrm{U}$ - Denotes the Number of Users:

$\mathrm{U}=\{\mathrm{u} 1, \mathrm{u} 2, \mathrm{u} 3 \ldots \ldots \ldots . . . \mathrm{un}\}$

$\mathrm{S}$ - Denotes the Number of Services:

$\mathrm{S}=\{\mathrm{s} 1, \mathrm{~s} 2, \mathrm{~s} 3 \ldots \ldots \ldots \ldots \mathrm{sn}\}$

Let Result for search of Hospital queries be Rh:

$\mathrm{Rh}=\mathrm{u} 1 \cap \mathrm{h} 1$

Let Result for search of Ambulance queries be Ra:

$\mathrm{Ra}=\mathrm{u} 1 \cap \mathrm{a} 1$

\section{Advantages}

$>$ It's beneficial for the users in case of emergencies as it saves time which gets consumed in searching for the ambulance by other means.

$>$ Information about the hospitals provided helps in getting the appropriate hospital which is suitable for the patient's treatment.

$>$ Sending patient's health information to the hospitals helps the hospital staff to get things ready required for the treatment.

$>$ Here the patients need not to wait in any case.

$>$ Hospitals information is directly provided through maps and hence there is no need to visit the particular hospital's website for information.

$>$ Live feeds will help for better a medical procedure which helps in saving patient's life in an effective way.

\section{Conclusion}

In this project, an idea is proposed for saving a patient's life in a faster way possible. It is beneficial for users in case of emergencies as it saves time. With this project, the ambulance can reach the patients as location is given through the control room and can provide necessary equipment required for the patient's health. Information about the hospitals provided helps in getting the appropriate hospital which is suitable for the patient's treatment. The live feed data sent through the ambulance to the hospital helps in keeping track of patient's health details and reach the hospital without any time lag. Sending patient's health information to the hospitals helps the hospital staff to get the necessary pre-requisites 
regarding the patient's treatment. Hence it reduces the time complexity and helps to provide faster medical services.

\section{References}

[1] Internet of things for Smart Cities. Andrea Zanella, Senior Member, IEEE, Nicola Bui, Angelo Castellani, Lorenzo Vangelista, Senior Member, IEEE, and Michele Zorzi, Fellow, IEEE. IEEE INTERNET OF THINGS JOURNAL, VOL. 1, NO. 1, FEBRUARY 2014.

[2] Veeramuthuvenkatesh, M.prashanthkumar, V.Vaithayanathan, Pethuru Raj," An ambient healthmonitor for the new generation healthcare," Journal of Theoretical and Applied Information Technology, Vol. 31 No.2, pp. 9199,Sep 2011.

[3] VeeramuthuVenkatesh,Pethuru Raj, KaushikGopalan and Rajeev.T," Healthcare Data Fusion and Presentation using Service-Oriented Architecture (SOA) Orchestration Mechanism," IJCA Special Issue on Artificial Intelligence Techniques - Novel Approaches \& Practical Applications, Vol. 2, pp. 17-23,June 2011

[4] Ruihua Zhang, and Dongfeng Yuan, "A Health Monitoring System for Wireless Sensor Networks," in Proc. of 2ed IEEE Conference on Industrial Electronics and Applications (ICIEA), pp. 1648-1652, Harbin, China, May 2007.

[5] Ruihua Zhang, and Dongfeng Yuan, "A Health Monitoring System for Wireless Sensor Networks," in Proc. of 2ed IEEE Conference on Industrial Electronics and Applications (ICIEA), pp. 1648-1652, Harbin, China, May 2007.

[6] Obuhuma, J. I., Moturi, C. A, "Use of GPS With Road Mapping For Traffic Analysis", IJSTR, vol 1, issue 10, Nov 2012

[7] Allwinkle, S. \& Cruickshank, P. (2011) Creating smarter cities: An overview, Journal of Urban Technology, 18(2), 1-16.

[8] Arshdeep Bahga, Vijay Madisetti, Internet of Things- A Hands on Approach, Universities Press, 2015.

[9] Emergency Management System Using Android Application RehkaJadhav, Jwalant Patel,Darshan Jain, Suyash Phadhtare Department of Information Technology G. H. Raisoni Collage of Engineering \& Technology, University of Pune, Pune.

[10] Ateeth Kumar Thirukkovulur1, Harikrishna Nandagopal, Vigneesh Parivallal," Intelligent Vehicle Control Based On Identification Of Road And Traffic Signal Operated RFID Transponders, International Conference On Advances In Electrical and Electronics Engineering (ICAEE'2012) Penang, Malaysia 
